\title{
Proposta de Uma Escala Portuguesa para a Avaliação da Qualidade do Aconselhamento Genético: Uma Nova Ferramenta para os Profissionais da Saúde
}

\section{Proposal of a Portuguese Tool for Quality Assessment of Genetic Counselling: a New Tool for Healthcare Professionals}

\author{
Milena PANEQUE* $\bigotimes^{1,2,3}$, Catarina COSTA $^{* 4}$, Carolina LEMOS ${ }^{1,2,5}$, Miguel ALVES-FERREIRA ${ }^{1,2,5}$, Jorge SEQUEIROS ${ }^{1,2,5}$, \\ Marina Serra LEMOS ${ }^{4}$ \\ Acta Med Port 2018 Jun;31(6):321-328 - https://doi.org/10.20344/amp.9997
}

\section{RESUMO}

Introdução: A falta de ferramentas para avaliar a qualidade do aconselhamento genético é uma limitação reconhecida em estudos nacionais e internacionais. É conhecida também a relação da qualidade da prática nos cuidados de saúde com uma maior satisfação dos doentes e das famílias afetadas. O seguinte estudo apresenta a construção e validação da primeira escala portuguesa para avaliação da qualidade da prática do aconselhamento genético. Engloba uma proposta de ferramenta para a avaliação do processo pelos próprios profissionais.

Material e Métodos: Iniciou-se este estudo pela revisão da literatura e identificação das principais dimensões do aconselhamento genético. De seguida, procedeu-se à elaboração dos itens e à sua organização mediante as dimensões teóricas do Modelo do Envolvimento Recíproco. Após um pré-teste alcançou-se a versão que foi proposta para validação a uma amostra de 30 participantes, que avaliaram 81 sessões de aconselhamento genético.

Resultados: Através de critérios estatísticos e empíricos selecionaram-se os melhores itens, ficando a escala constituída por 50 itens. Esta versão da escala compreende cinco dimensões: educação, características do consultando e tomada de decisão, relação terapêutica, efeitos do processo no consultando e organização do serviço.

Discussão: Os resultados mostraram que se trata de uma escala válida, com características psicométricas consistentes e fundamentada em simultâneo do ponto de vista teórico-prático do aconselhamento genético. O número reduzido de participantes envolvidos na validação da escala constitui uma limitação, que reflete o número reduzido de profissionais a exercer nesta área dos cuidados de saúde.

Conclusão: A escala proposta neste estudo é um instrumento pioneiro, multidimensional que pretende contribuir para a qualidade da prática do aconselhamento genético em Portugal.

Palavras-chave: Aconselhamento Genético; Avaliação de Resultados (Cuidados de Saúde); Portugal; Psicometria; Serviços de Genética

\section{ABSTRACT}

Introduction: The lack of tools for quality assessment of genetic counselling is recognized in national and international studies. The correlation of quality of healthcare practice with greater satisfaction of patients and affected families is also well established. The present study describes the development and validation of the first Portuguese scale for quality assessment of genetic counselling practice. It encompasses a proposal of a new tool for the evaluation of the process by professionals.

Material and Methods: The definition of an initial pool of items and their organization was based on a literature review and identification of the main genetic counselling dimensions as well as the theoretical dimensions of the Reciprocal Engagement Model. After a pre-test validation the scale was submitted to psychometric validation using a sample of 30 participants who evaluated 81 genetic counselling sessions.

Results: Based on statistical and empirical criteria the best items were selected. The final 50 items- version comprises five dimensions: education, counselees' characteristics and decision-making, therapeutic relationship, effects of the process on the counselees and services organization.

Discussion: Results showed consistent psychometric properties of the scale supported on theoretical and practice concepts of genetic counseling. The reduced number of participants involved in psychometric validation is a limitation of the study, which reflected the reduced number of professionals in genetic healthcare services.

Conclusion: The scale proposed at this study is a novel and multidimensional instrument that aimed to contribute to the improvement of genetic counselling practice in Portugal.

Keywords: Genetic Counseling; Genetic Services; Outcome Assessment (Health Care); Portugal; Psychometrics

\footnotetext{
Os dois primeiros autores deste trabalho contribuíram de forma equitativa

1. i3S - Instituto de Investigação e Inovação em Saúde. Universidade do Porto. Porto. Portugal.

2. IBMC - Instituto de Biología Molecular e Celular. Universidade do Porto. Porto. Portugal.

3. CGPP - Centro de Genética Preditiva e Preventiva. Universidade do Porto. Porto. Portugal.

4. FPCEUP - Faculdade de Psicologia e de Ciências da Educação. Universidade do Porto. Porto. Portugal.

5. ICBAS - Instituto de Ciências Biomédicas Abel Salazar. Universidade do Porto. Portugal.

$\square$ Autor correspondente: Milena Paneque. milenaph@ibmc.up.pt

Recebido: 30 de novembro de 2017 - Aceite: 03 de maio de 2018 | Copyright ( O Ordem dos Médicos 2018
} 


\section{INTRODUÇÃO}

O aconselhamento genético é uma área interdisciplinar que tem vindo a afirmar-se como uma nova profissão nos cuidados de saúde, particularmente dos serviços de genética. ${ }^{1}$ Internacionalmente, e especialmente na Europa, tem-se assistido a um crescente reconhecimento da imprescindibilidade de ser oferecido aconselhamento genético aquando a realização de testes genéticos, como base de uma prática segura e da tomada de decisões informadas pelos utentes. ${ }^{2,3}$

Em Portugal, o aconselhamento genético é efetuado pelos médicos especialistas em genética médica desde que existe esta especialização e o seu desenvolvimento esteve principalmente associado à implementação do Protocolo de Testes Preditivos para as principais doenças neurológicas de início tardio, que possuem uma prevalência particularmente elevada no nosso país. ${ }^{4,5} \mathrm{Em}$ meados dos anos 90 foi assim definida a necessidade de oferecer aconselhamento genético aos indivíduos em risco para estas condições genéticas, concebendo como elementos chave do protocolo de consultas o suporte informativo, a tomada de decisão e o apoio psicossocial. A genética preditiva e a possibilidade de diagnosticar, de forma antecipada o início dos sintomas clínicos, a presença das mutações causadoras destas doenças incuráveis foi, talvez por isso, um contexto único de aprendizagem e de aquisição de competências para os profissionais dos serviços de genética no país. Apesar de existir uma via académica, a nível de mestrado profissionalizante para a formação das competências específicas do aconselhamento genético para profissionais não médicos, esta é ainda uma profissão não reconhecida em Portugal. ${ }^{5}$

Nos últimos anos tem proliferado a investigação nacional na área do aconselhamento e da genética psicossocial como fonte para a melhoria da prática clínica nos cuidados de saúde. ${ }^{6-11}$ As atuais potencialidades e constrangimentos relativamente à área da genética tanto nos cuidados de saúde primários como nos próprios serviços especializados em genética têm sido alvo da atenção de clínicos, investigadores e entidades reguladoras de saúde. $5,8,12,13 \mathrm{~A}$ falta de recursos humanos e a necessidade de formação de competências específicas destacam-se entre as limitações principais, pelo que as consultas de aconselhamento genético têm sido principalmente asseguradas pelos médicos geneticistas, neurologistas e obstetras, existindo tempos de espera elevados para o acesso a alguns serviços de genética. ${ }^{13}$ No que refere à qualidade dos serviços que são prestados, um recente estudo nacional evidenciava como a generalidade dos profissionais dos cuidados de saúde de genética percebia limitações na definição dos indicadores de efetividade e competência e a ausência de diretrizes nacionais para uma prática adequada do aconselhamento genético. ${ }^{12}$ Este cenário não é exclusivo do nosso país. ${ }^{14}$

De modo geral, as ferramentas disponíveis para a avaliação da qualidade do aconselhamento genético são reduzidas ou pouco divulgadas internacionalmente ${ }^{15-19}$ sendo os métodos mais utilizados focados nos possíveis efeitos do aconselhamento ${ }^{20}$ e não nas componentes do processo que garantem a sua eficácia e a sua utilidade para os pacientes. ${ }^{16,21}$ Das poucas escalas que têm sido desenvolvidas no âmbito do aconselhamento genético é ainda de realçar o facto de todas serem focadas em áreas muito específicas do mesmo e nenhuma delas ter sido ainda adaptada ao contexto português. ${ }^{19,22,23} \mathrm{De}$ facto, tradicionalmente têm vindo a ser utilizadas escalas que avaliam separadamente, entre outros aspetos, a satisfação, o conhecimento, a tomada de decisão, as estratégias de coping dos pacientes, a comunicação familiar sobre a doença e sobre o risco. ${ }^{15,23}$ A maioria destas ferramentas resultam de instrumentos da psicologia ou da medicina adaptadas ao aconselhamento genético pelo que nenhuma é ainda suficientemente abrangente para a avaliação integral dos aspetos relevantes da sua qualidade, do seu processo e dos seus potenciais efeitos. ${ }^{15,23} \mathrm{~A}$ inexistência de escalas de avaliação fundamentadas concetualmente e validadas empiricamente impede uma adequada auditoria dos serviços, o estudo das suas limitações e potencialidades e a comparação dos distintos modelos de prestação de cuidados. ${ }^{24,25}$ Por este motivo, a criação de ferramentas para os profissionais avaliarem a sua prática clínica e medirem os parâmetros de competência é per se um contributo para o melhoramento dos serviços. ${ }^{14}$

Surgiu assim a necessidade de desenvolver uma escala para avaliação da prática do aconselhamento genético em Portugal, que emergisse do contexto atual dos cuidados de saúde e que fosse capaz de dar resposta às lacunas anteriormente expostas. O presente artigo descreve, assim, o processo de construção e validação da primeira escala portuguesa para a avaliação da qualidade da prática do aconselhamento genético. É objetivo deste artigo também, a descrição das suas propriedades psicométricas tais como a análise das componentes principais, da consistência interna e das relações entre as dimensões avaliadas pela escala.

\section{MATERIAL E MÉTODOS \\ O primeiro passo metodológico: a procura do modelo teórico-prático da escala}

Sendo útil escolher um modelo teórico para orientar a configuração da estrutura de base de uma escala, a primeira fase deste estudo esteve centrada na procura de um modelo teórico apropriado. Um modelo teórico-prático permite também a definição dos princípios e dos objetivos, neste caso do aconselhamento, que podem por sua vez, servir de guia para a identificação das dimensões do aconselhamento genético a serem estudadas. ${ }^{20}$

Historicamente, o aconselhamento genético baseou-se em modelos da medicina, da educação e da saúde mental. ${ }^{26,27}$ Tal como citado por McCarthy Veach et al (2003), nos primórdios, Sheldon Reed e mais tarde, Joan Marks consideraram o aconselhamento como uma forma de genética social, com o objetivo de apresentarem uma abordagem psicossocial das condições genéticas. ${ }^{27}$ 
Esta abordagem visava reconhecer que os consultandos existem dentro de um sistema familiar e social, não fazendo sentido avaliá-los como uma entidade no vácuo. Posteriormente, Kessler (1997) descreveu o modelo de ensino e o modelo de aconselhamento como as duas abordagens dos profissionais na prática do aconselhamento genético. ${ }^{28,29} \mathrm{O}$ modelo de ensino, importado da medicina, tinha como principal objetivo fornecer informações ao consultando para ele tomar as suas próprias decisões. Por sua vez, o modelo de aconselhamento, proveniente da área da saúde mental, estruturava-se na compreensão do outro e numa prática centrada no paciente. ${ }^{29}$

Mais recentemente, numa tentativa de definir pela primeira vez um modelo próprio do aconselhamento genético, foi proposto o Modelo do Envolvimento Recíproco ${ }^{28}$ que engloba contributos de todos os modelos anteriores e que foi escolhido para ser o modelo teórico adjacente à escala construída neste estudo (Fig. 1).

Este modelo surgiu de uma tentativa de normalização da prática por parte de um conjunto de profissionais de longa experiência na área do aconselhamento genético, sendo os princípios de base do modelo os seguintes: a informação é fundamental, o relacionamento é parte integrante do aconselhamento, a autonomia deve ser apoiada, os consultandos são resilientes e as suas emoções fazem a diferença. ${ }^{28} \mathrm{~A}$ cada princípio do modelo são associados distintos objetivos, estratégias e comportamentos específicos de modo a se alcançarem os resultados pretendidos (Apêndice 1: https://www.actamedicaportuguesa.com/revista/index.php/amp/article/view/9997/5454).

Tanto quanto conhecemos, a escala que aqui se reporta é a primeira experiência de utilização deste modelo como referencial teórico-prático de base para a construção de uma ferramenta de avaliação da prática.

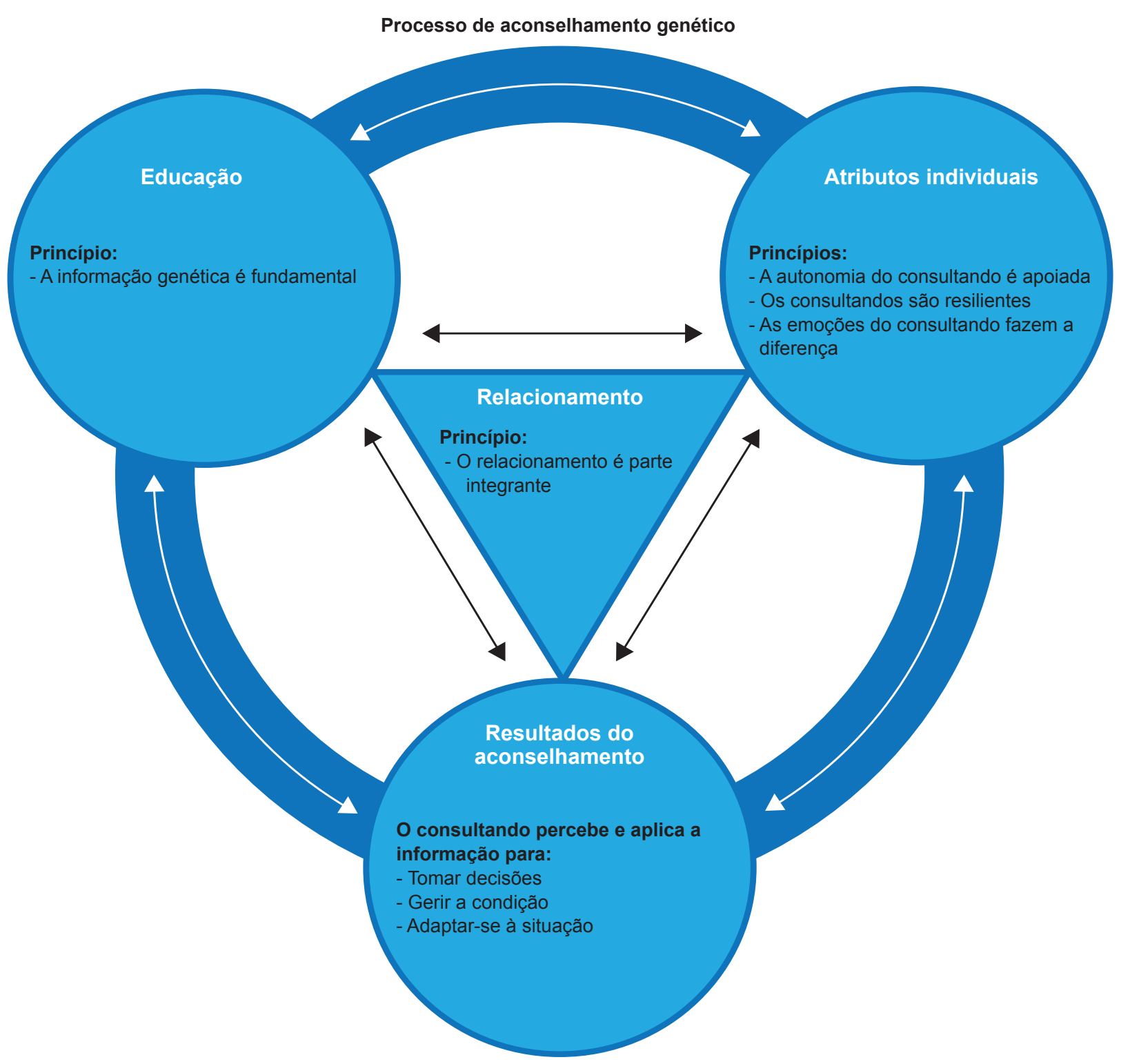

Figura 1 - Esquematização das dimensões e dos princípios do Modelo do Envolvimento Recíproco ${ }^{28}$

Cada elemento interage com todos os outros elementos. Nenhum permanece sozinho ou trabalha isoladamente. 
Validação da escala: procedimentos de pré-teste e validade de conteúdo

A primeira formulação dos itens resultou de pesquisas bibliográficas para a identificação das principais dimensões e a forma como tinham sido reportadas algumas destas temáticas na literatura. Complementarmente, efetuámos a análise de estudos nacionais sobre a perceção dos profissionais e dos consultandos acerca dos critérios relevantes para uma prática com qualidade. ${ }^{11,12,21,25}$ Estes estudos qualitativos prévios serviram como validação cultural dos indicadores de qualidade do aconselhamento genético na população portuguesa (aconselhadores e consultandos), emergindo assim do contexto atual dos cuidados de saúde em Portugal a primeira versão da escala.

De seguida, definimos o formato da escala e as opções de resposta. Submetemos esta primeira versão a um pré-teste exploratório junto de peritos da área. Para esta validação pré-teste da escala inicial utilizámos a metodologia da entrevista cognitiva ${ }^{30}$ envolvendo um painel de cinco peritos (dois médicos geneticistas, dois aconselhadores genéticos e um psicólogo). A entrevista cognitiva é um processo de administração de perguntas com o objetivo de obter informação adicional acerca do significado das questões de uma escala e de compreender o processo de pensamento do entrevistado. ${ }^{31}$

De modo geral, foi proposta a avaliação dos itens quanto a critérios tais como a formulação objetiva, a simplicidade, a relevância, a amplitude, a clareza, as alternativas de resposta, as ambiguidades e as dificuldades sentidas. No seu todo, a escala sofreu análise relativamente à suficiência das instruções, à deteção de itens mal construídos, à sensibilidade das questões e dos itens, à qualidade das alternativas de resposta, ao alcance do conteúdo e à relevância para a avaliação da prática. ${ }^{32}$ Obtidas as respostas dos peritos, o conteúdo foi analisado tendo em conta o contexto português e as evidências da prática clínica.

\section{Validação psicométrica: procedimento de recolha de dados}

Com o intuito de dar seguimento à validação da escala construída, elaborámos uma listagem com todos os serviços em Portugal que disponibilizam consultas de aconseIhamento genético. De seguida, contactámos os serviços via e-mail e visitámos os mesmos após aceitação da participação na investigação, fornecendo-se cópias da escala, uma folha informativa do estudo, os seus objetivos e as questões éticas da confidencialidade dos dados.

Apenas foram elegíveis para participar os profissionais a realizar consultas de aconselhamento genético: médicos Geneticistas, Oncogeneticistas e aconselhadores genéticos. Devido à população-alvo ser pequena e ser necessária uma credível análise estatística, propusemos a cada profissional o preenchimento por três vezes da escala, focando-se em três sessões. Junto de cada conjunto de escalas disponibilizámos um envelope selado para que fosse possível ao profissional lacrar e enviar para o nosso serviço.

\section{Análise de Dados}

Efetuámos a análise de dados com recurso ao software SPSS versão 24, incluindo a análise das componentes principais, da consistência interna dos itens e das correlações entre os itens, para a validação da escala construída, considerando um nível de significância de 0,05.

\section{RESULTADOS}

\section{Primeira versão da escala}

A escala elaborada ficou composta por cinco blocos de questões relativos à avaliação da qualidade da prática, perfazendo um conjunto de 75 itens que representam aspetos e dimensões importantes para a prática do aconselhamento genético. Os itens foram organizados à luz do Modelo do Envolvimento Recíproco e abrangendo as suas dimensões. O primeiro conjunto de questões diz respeito ao princípio da relevância da informação na prática do aconselhamento genético, pertencente à dimensão de 'Educação'. O segundo conjunto relaciona-se com a abordagem das questões emocionais e motivacionais, as características individuais dos consultandos e o suporte na tomada de decisões, referentes à dimensão dos 'Atributos Individuais' do modelo. $\mathrm{O}$ terceiro conjunto de questões aborda questões relacionais e de comunicação do aconselhamento genético, sobre o princípio de que as preocupações e dúvidas dos consultandos são abordadas de forma mais efetiva quanto mais forte, empática e respeitadora da autonomia do consultando for a relação entre o profissional e o consultando para dar resposta às questões médicas, educacionais e psicossociais envolvidas, que se encontra enquadrado na dimensão da 'Relação'. O quarto bloco de questões incide sobre os possíveis efeitos do aconselhamento no consultando, que estão relacionados com a dimensão dos 'Resultados'. Por fim, o quinto conjunto de itens aborda o 'Funcionamento' dos serviços, que embora não faça parte do modelo se considera um tema essencial para a avaliação da prática no seu todo.

Para além disso, foram recolhidos como dados sociodemográficos dos profissionais a idade e o género. A escala de resposta é do tipo Likert, de cinco pontos, em que 1 corresponde a um nível muito baixo do aspeto avaliado e 5 a um nível muito elevado, existindo ainda uma opção para a resposta de 'não se aplica' (tendo em conta a diversidade de tipologias de consultas de aconselhamento genético).

No pré-teste, a análise das entrevistas cognitivas permitiu organizar um conjunto de linhas orientadoras na reformulação da escala. Os peritos avaliaram as questões segundo a sua adequação para a população-alvo ou contexto cultural, a formulação objetiva dos itens, a consistência em certas palavras ou frases, o grau de compreensão, as potenciais redundâncias entre outros aspetos. Como resultado deste procedimento metodológico, introduziram-se algumas alterações na formulação de palavras e expressões, bem como no formato de apresentação de alguns itens. Alguns itens foram eliminados e outros adicionados mantendo-se um total de 74 itens. 


\section{Validação psicométrica da escala Participantes}

Existem atualmente, sete serviços de genética em Portugal e ainda outras 10 entidades hospitalares a efetuarem aconselhamento genético. Deste conjunto, cinco serviços de genética aceitaram participar neste estudo, bem como quatro entidades que oferecem aconselhamento genético e que serviram para reforçar o reduzido número de profissionais existente nos serviços de genética. A amostra desta investigação é constituída, assim, por 30 participantes de oito instituições hospitalares. As idades dos participantes variam entre os 26 e os 60 anos ( $M=39,38$; $D P=10,38)$, sendo que 20 são do género feminino $(66,7 \%)$ e 10 do género masculino $(33,3 \%)$.

A generalidade dos serviços e dos profissionais demonstrou recetividade e muito interesse perante o estudo e a escala construída, revelando uma certa curiosidade acerca do modelo teórico adjacente à elaboração da mesma. Essa aceitação positiva foi verificada não só aquando das deslocações aos serviços de genética do nosso país como também nas reuniões nacionais e internacionais nas quais apresentámos o trabalho desenvolvido.

\section{Análise das componentes principais e da consistência interna}

Nesta fase do estudo, optámos por fazer a análise separada de cada dimensão. Note-se que as três primeiras dimensões se referem a critérios que devem ser contemplados na prática do aconselhamento genético como a relevância da informação, a abordagem das questões emocionais e características individuais bem como aspetos relacionais e de comunicação. A quarta e quinta dimensões são aspetos distintos, a primeira abordando os possíveis efeitos do aconselhamento genético no consultando e a segunda apresentando os indicadores de funcionamento dos serviços. Assim, a escala não pretende fornecer uma medida global destes aspetos que são, de facto, distintos.

Para verificar as componentes principais, efetuámos a análise da adequabilidade da amostra e da capacidade de rotação dos fatores, através do cálculo do coeficiente de Kaiser-Meyer-Olkin (KMO) e do teste da esfericidade de Bartlett. Os valores de $\mathrm{KMO}$ indicam que existe uma adequabilidade da amostra para a análise em componentes principais em todas as dimensões, uma vez que os valores são superiores a 0,50 $(0,57 \leq \mathrm{KMO}$ de todas as dimensões $\leq 0,86$ ). No teste da esfericidade obtivemos um valor inferior a 0,001 em todas as dimensões, o que indica uma boa correlação entre os itens de cada uma delas. ${ }^{33}$

Recorremos à análise das componentes principais de cada dimensão, procedendo a uma análise exploratória sem predefinição do número de fatores. Esta técnica foi efetuada para explorar as inter-relações entre os dados das variáveis e se os mesmos têm o mesmo conceito/fator subjacente. $\mathrm{Na}$ análise das componentes principais, optou-se pela rotação oblíqua (método Oblimin) com normalização de Kaiser (Apêndice 2: https://www.actamedicaportuguesa.com/revista/index.php/amp/article/view/9997/5455). Foi tido também em consideração que cada dimensão tinha fatores correlacionados, o que parece mais característico da área da psicologia dada a interdependência das dimensões psicológicas. $^{32}$

Deste modo, no sentido de confirmarmos a estrutura da escala e procedermos a uma redução dos itens tivemos em conta a análise em componentes principais (Apêndice 2) nos fatores com valor próprio superior a 1 e os valores de saturação iguais ou superiores a 0,40. ${ }^{34}$ Utilizámos também a análise das correlações entre itens, bem como a relevância teórica e prática de cada um deles.

A consistência interna de cada dimensão foi avaliada através do cálculo do coeficiente $\alpha$ de Cronbach.
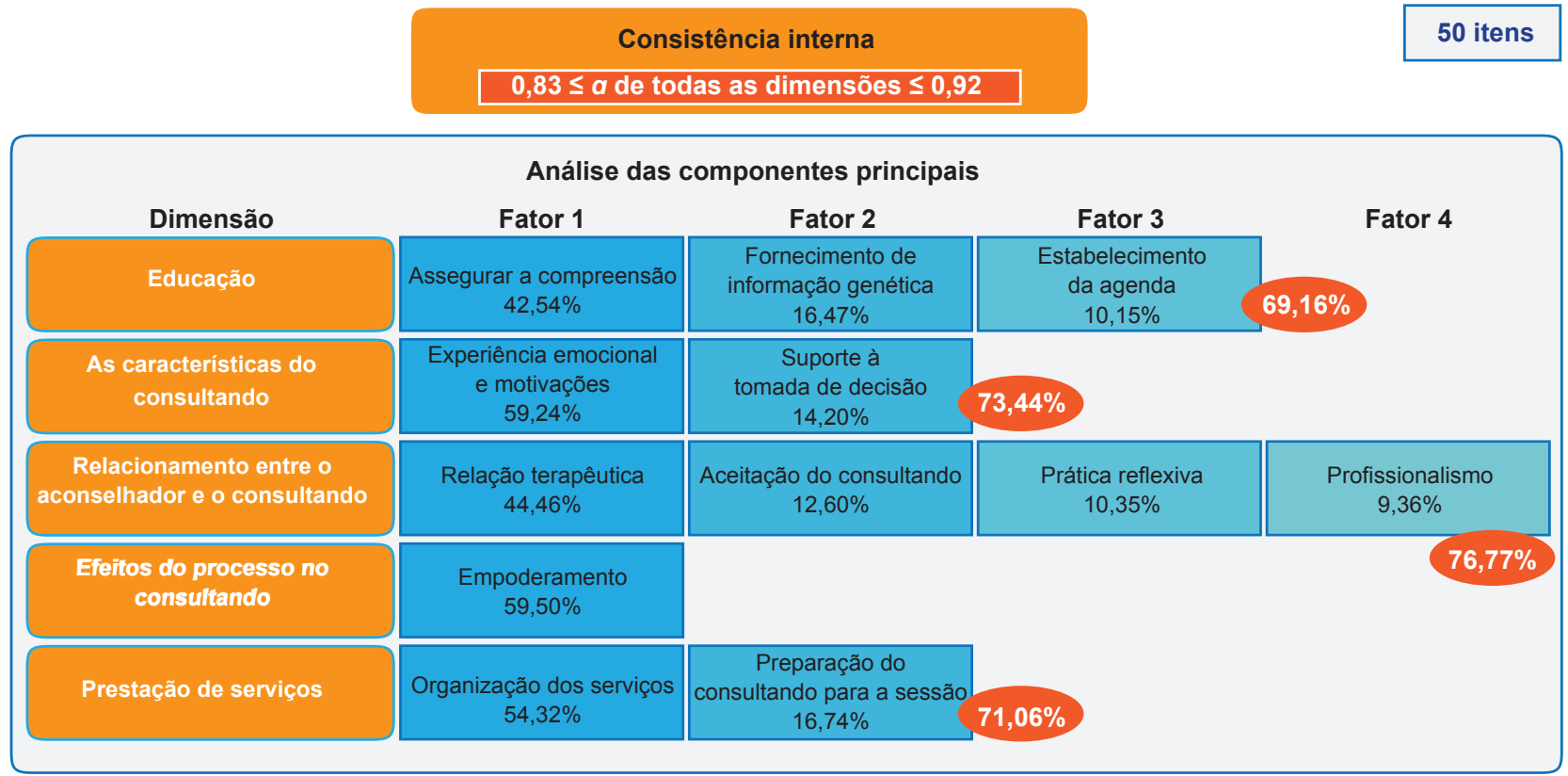

Figura 2 - Propriedades psicométricas da versão final da escala proposta 
Na versão inicial de 74 itens, todas as dimensões da escala apresentaram boa consistência interna (valores do $\alpha$ de Cronbach entre 0,85 e 0,96) e bons resultados na análise dimensional (valores de variância cumulativa explicada entre $67,11 \%$ e $78,81 \%$ ), contudo reduzimos o número de itens para uma melhor funcionalidade da escala na prática. Todos os itens apresentaram nos diferentes fatores uma saturação superior a 0,40 , aspeto que garante uma boa comunalidade das afirmações construídas. ${ }^{34,35} \mathrm{~A}$ análise das componentes principais determinou a exclusão de dois itens por saturarem em dois fatores distintos. Através da análise de correlações e da relevância teórica, eliminámos outros 22 itens, perfazendo um total de 24 itens eliminados.

\section{A versão final da escala e a sua cotação}

A versão reduzida da escala é constituída por 50 itens (Apêndice 3: https://www.actamedicaportuguesa.com/ revista/index.php/amp/article/view/9997/5456) e cinco dimensões que mostraram bons valores de $\alpha$ de Cronbach, entre os 0,83 e 0,92, o que indica uma boa consistência interna da ferramenta (Fig. 2). Os fatores de cada dimensão explicaram uma variância cumulativa considerável, entre $59,50 \%$ e $76,77 \%$, o que garante uma boa validade de constructo.

A cotação proposta para análise das respostas do preenchimento da escala é baseada na análise estatística dos scores de cada dimensão. Para tal, definiu-se como procedimento de cálculo o seguinte: 1) a obtenção da mediana para cada fator de cada dimensão (pois o valor da média é muito afetado pelas pontuações extremas); 2) a multiplicação da mediana de cada fator pela sua percentagem de variância explicada na análise das componentes principais (de forma a considerar o peso que cada fator tem dentro de cada dimensão); 3) a normalização das percentagens para $100 \%$ (pois cada dimensão nunca tem uma variância explicada acumulada de 100\%); 4) o somatório destas percentagens normalizadas para obter a percentagem total de cada dimensão. A classificação dos valores finais é a seguinte: valores inferiores a $20 \%$ são conside- rados como uma avaliação fraca da qualidade da prática dos aspectos incluídos nessa dimensão; valores entre $21 \%$ e $40 \%$ são considerados como uma avaliação insuficiente; valores entre $41 \%$ e $60 \%$ são considerados como uma avaliação suficiente; valores entre $61 \%$ e $80 \%$ são considerados como uma avaliação boa; e valores iguais ou superiores a $81 \%$ são considerados como uma avaliação muito boa da prática do aconselhamento genético que teve lugar na sessão avaliada.

Os valores obtidos na cotação das dimensões da escala no processo de validação psicométrica deste estudo nacional podem servir como valores de referência e estão ilustrados na Tabela 1. De modo a simplificar a obtenção destes valores pelos utilizadores construímos uma fórmula de cálculo base.

\section{DISCUSSÃO}

A escala desenvolvida encontra-se bem fundamentada não só devido à sua justificação teórica mas também devido à procura de concetualização das dimensões e respetivas subdimensões, articulando os aspetos empíricos, teóricos e práticos subjacentes à investigação nacional desenvolvida pelos autores da mesma. ${ }^{11,12,21,25} \mathrm{O}$ instrumento construído nesta investigação demonstrou propriedades psicométricas apropriadas, o que possibilita a sua utilização imediata no contexto clínico e de investigação. Com base nos resultados obtidos, podemos afirmar que a escala resultante deste estudo é um instrumento apto para ser utilizado pelos profissionais de saúde. A escala difere de outras ferramentas construídas para a avaliação da qualidade do aconselhamento genético disponibilizadas aos pacientes ${ }^{18,19}$ e que se focam em aspetos específicos do processo e/ou os seus efeitos. ${ }^{16-20}$

Salienta-se que a cotação das distintas dimensões da escala obtidas pelos participantes neste estudo pode servir como valores de referência para futuros estudos nacionais em relação a componentes tão relevantes dos cuidados de saúde tais como: a organização dos serviços; a existência de uma prática reflexiva por parte dos profissionais;

Tabela 1 - Valores de referência da cotação das dimensões e fatores da escala

\begin{tabular}{|c|c|c|c|c|c|}
\hline \multicolumn{6}{|l|}{ Medianas } \\
\hline & Dimensão 1 & Dimensão 2 & Dimensão 3 & Dimensão 4 & Dimensão 5 \\
\hline Fator 1 & $\begin{array}{l}\text { Itens } 1.5,1.6,1.9 \\
1.10 \text { e } 1.11 \\
\mathbf{3 , 0 0}\end{array}$ & $\begin{array}{l}\text { Itens } 2.1 \text { a } 2.7 \\
4,00\end{array}$ & $\begin{array}{l}\text { Itens } 3.1,3.2,3.3,3.7 \\
\text { e } 3.8 \\
\mathbf{4 , 0 0}\end{array}$ & $\begin{array}{l}\text { Itens } 4.1 \text { a } 4.8 \\
\mathbf{5 , 0 0}\end{array}$ & $\begin{array}{l}\text { Itens } 5.1, \quad 5.3, \\
5.5,5.6 \text { e } 5.7 \\
\mathbf{4 , 5 0}\end{array}$ \\
\hline Fator 2 & $\begin{array}{l}\text { Itens } 1.2,1.3,1.4 \\
1.7,1.8 \text { e } 1.12 \\
\mathbf{5 , 0 0}\end{array}$ & $\begin{array}{l}\text { Itens } 2.8 \text { a } 2.11 \\
5.00\end{array}$ & $\begin{array}{l}\text { Itens } 3.3 \text { a } 3.5 \\
\mathbf{5 , 0 0}\end{array}$ & & $\begin{array}{l}\text { Itens } 5.2,5.3,5.4 \\
\text { e } 5.8 \\
\mathbf{4 , 5 0}\end{array}$ \\
\hline Fator 3 & $\begin{array}{l}\text { Itens } 1.1 \text { e } 1.12 \\
\mathbf{4 , 5 0}\end{array}$ & & $\begin{array}{l}\text { Itens } 3.10 \text { e } 3.11 \\
3,00\end{array}$ & & \\
\hline Fator 4 & & & $\begin{array}{l}\text { Itens } 3.6 \text { e } 3.9 \\
\mathbf{4 , 5 0}\end{array}$ & & \\
\hline Percentag & da dimensão (\%) & & & & \\
\hline & 73,93 & 83,87 & 81,80 & 100,00 & 90,00 \\
\hline
\end{tabular}


a qualidade do suporte informativo e a garantia da compreensão da informação médica que é oferecida; a identificação das necessidades dos pacientes e o reconhecimento do valor das suas características individuais para a adequação do processo de consulta; o suporte na tomada de decisões e o empoderamento dos pacientes; a antecipação dos potenciais efeitos do aconselhamento genético e a existência de uma relação terapêutica forte, empática e respeitadora da autonomia do consultando.

Este estudo poderá comportar algumas limitações. É preponderante salientar que o número de especialistas no ativo a exercer aconselhamento genético em Portugal é reduzido, o que se mostrou uma limitação ao nível da análise estatística dos dados. Este aspeto foi agravado pelo facto de os especialistas participantes nem sempre responderem aos três questionários pretendidos, o que reduziu o número final de questionários obtidos. Contudo, dos 58 profissionais a exercer aconselhamento genético no nosso país tivemos uma participação de 30 sujeitos (52\%) e, tal como foi referido anteriormente, a aceitação positiva da criação da escala foi reflexo da perceção quanto à sua utilidade para os serviços, explicando a recetividade deste estudo pelos principais serviços do país.

A necessidade de preenchimento da escala após três consultas distintas não possibilitou o levantamento imediato das respostas individuais e talvez por isso possa ter diminuído o número de participantes. Para colmatar a limitação do número de participantes e aprofundar a validação psicométrica, no futuro, podem ser incluídos estudos de análises fatoriais confirmatórias propondo uma nova organização dos itens e estudos de invariância métrica para a avaliação da estabilidade da estrutura da escala, entre outras.

Contudo, é de salientar que o instrumento sofreu uma validação preliminar satisfatória e que mais evidências empíricas permitirão um melhor afinamento da escala atual. Assim sendo, vários estudos podem ser necessários futuramente para explorar melhor as características da escala, sendo importante replicar as análises efetuadas neste estudo com uma amostra maior de participantes que possibilite a confirmação dos resultados obtidos e a redução de possíveis disparidades. Esta escala poderá ser também aplicada a profissionais de outros países para se verificar a validação e a aplicabilidade internacional da mesma. De facto, já foi verificado interesse para tal, por parte de profissionais do aconselhamento genético da Noruega, Espanha e França. ${ }^{36}$

\section{CONCLUSÃO}

Esta proposta de escala, pioneira no seu género visa conduzir o profissional que realiza aconselhamento genético na reflexão sobre a sua prática clínica, funcionando como uma espécie de guia orientador da prática profissional. A escala desenvolvida é um instrumento multidimensional que traz importantes contributos para a prática do aconselhamento genético em Portugal. Aparenta ser bastante útil na prática reflexiva dos profissionais e na avaliação dos serviços prestados na área da genética.

A proposta de escala avalia indicadores relevantes da relação entre o profissional e o consultando para dar resposta às questões médicas, educacionais e psicossociais envolvidas no processo de aconselhamento genético e de tomada de decisões nos cuidados de saúde. Apesar de ser apenas centrada nos profissionais, permite aos mesmos verificar se cumpriram os objetivos determinados para cada sessão, sendo esta uma medida da utilidade da ferramenta. Assim, poderá ser relevante na avaliação da qualidade dos serviços em genética, identificando aspetos menos desenvolvidos e permitindo a criação de programas de intervenção oportunos.

\section{AGRADECIMENTOS}

Aos profissionais e às instituições que colaboraram com este trabalho, Hospital de Santa Maria, Hospital Pediátrico de Coimbra, Instituto Português de Oncologia do Porto, Hospital de São João, Hospital de Braga, Hospital de Santo António, Hospital Divino Espírito Santo, Hospital de Faro. Agradece-se também a Diogo Pereira e Miguel Sousa pelo apoio na análise dos dados e na definição dos procedimentos de cotação da escala.

\section{PROTECÇÃO DE PESSOAS E ANIMAIS:}

Os autores declaram que os procedimentos seguidos estavam de acordo com os regulamentos estabelecidos pelos responsáveis da Comissão de Investigação Clínica e Ética e de acordo com a Declaração de Helsínquia da Associação Médica Mundial.

\section{CONFIDENCIALIDADE DOS DADOS:}

Os autores declaram ter seguido os protocolos do seu centro de trabalho acerca da publicação de dados.

\section{CONFLITOS DE INTERESSE:}

Os autores declaram não terem qualquer conflito de interesse relativamente ao presente artigo.

\section{FONTES DE FINANCIAMENTO:}

Os autores declaram não ter recebido subsídios ou bolsas para a elaboração do artigo.

\section{REFERÊNCIAS}

1. Paneque M, Moldovan R, Cordier C, Serra-Juhé C, Feroce I, Bjørnevoll I, et al. Genetic counselling orofession in Europe. Chichester: John Wiley \& Sons, Ltd; 2016.

2. Kääriäinen $H$, Hietala $M$, Kristoffersson $U$, Nippert I, Rantanen $E$, Sequeiros J, et al. Recommendations for genetic counselling related

to genetic testing. Belgium: Eurogentest. [consultado 2017 Ago 14] Disponível em: http://www.eurogentest.org/index.php?id=674.

3. Skirton H, Goldsmith L, Jackson L, Tibben A. Quality in genetic counseling for presymptomatic testing - clinical guidelines for practice across the range of genetic conditions. Eur J Hum Genet. 2013;21:256- 
60.

4. Sequeiros J. O teste preditivo da doença de Machado-Joseph. Porto: UnIGENe, IBMC; 1996

5. Paneque M, Mendes À, Saraiva J, Sequeiros J. Genetic counseling in Portugal: education, practice and a developing profession. J Genet Couns. 2015a;24:548-52

6. Rolim L, Leite Â, Lêdo S, Paneque $M$, Sequeiros J, Fleming $M$. Psychological aspects of pre-symptomatic testing for MachadoJoseph disease and familial amyloid polyneuropathy type I. Clin Genet. 2006;69:297-305.

7. Paneque $M$, Lemos $C$, Sousa $A$, Velázquez L, Fleming $M$, Sequeiros J. Role of the disease in the psychological impact of pre-symptomatic testing for SCA2 and FAP ATTRV30M: experience with the disease, kinship and gender of the transmitting parent. J Genet Couns. 2009;18:483-93.

8. Mendes Á, Sousa L, Paneque M. From constraints to opportunities? Provision of psychosocial support in Portuguese oncogenetic counseling services. J Genet Couns. 2013;22:771-83.

9. Mendes A, Paneque M, Sousa L, Clarke A, Sequeiros J. How communication of genetic information within the family is addressed in genetic counselling: a systematic review of research evidence. Eur J Hum Genet. 2016;24:315-25.

10. Lêdo S, Paneque M, Rocha JC, Leite Â, Sequeiros J. Predictive testing for two neurodegenerative disorders (FAP and HD): a psychological point of view. Open J Genet. 2013:3:270-9.

11. Guimarães L, Sequeiros J, Skirton H, Paneque M. What counts as effective genetic counselling for presymptomatic testing in late-onset disorders? A study of the consultand's perspective. J Genet Couns. 2013;22:437-47.

12. Paneque M, Mendes À, Guimarães L, Sequeiros J, Skirton H. Genetics health professionals' views on quality of genetic counseling service provision for presymptomatic testing in late-onset neurological diseases in Portugal: core components, specific challenges and the need for assessment tools. J Genet Couns. 2015b;24,616-25.

13. Magalhães S, Paneque M, Silva J. Genetics on primary healthcare: a multidisciplinary perspective. Acta Med Port. 2016;29:581-2.

14. McAllister M, Moldovan R, Paneque M, Skirton $H$. The need to develop an evidence base for genetic counselling in Europe. Eur J Hum Genet. 2016;24:504-5.

15. Bernhardt BA, Biesecker BB, Mastromarino CL. Goals, benefits, and outcomes of genetic counseling: Client and genetic counselor assessment. Am J Med Genet. 2000;94:189-97.

16. Biesecker BB, Peters KF. Process studies in genetic counseling: peering into the black box. Am J Med Genet. 2001;106:191-8.

17. Payne K, Nicholls S, McAllister M, MacLeod R, Donnai D, Davis L. Outcome measurement in clinical genetics services: a systematic review of validated measures. Value Health. 2008;11:497-508.

18. Skirton $\mathrm{H}$, Parsons $\mathrm{E}$, Ewings $\mathrm{P}$. Development of an audit tool for genetic services. Am J Med Genet. 2005;136A:122-7.

19. McAllister M, Wood AM, Dunn G, Shiloh S, Tood C. The Genetic
Counseling Outcome Scale: a new patient-reported outcome measure for clinical genetics services. Clin Genet. 2011;79:413-24.

20. Wang C, Gonzalez R, Merajver SD. Assessment of genetic testing and related counseling services: current research and future directions. J Sports Sci Med. 2004;58:1427-42.

21. Paneque M, Sequeiros J, Skirton H. Quality assessment of genetic counseling process in the context of presymptomatic testing for lateonset disorders: a thematic analysis of three review articles. Genet Test Mol Biomarkers. 2012;16:36-45.

22. Meiser B, Irle J, Lobb E, Barlow-Stewart K. Assessment of the content and process of genetic counseling: a critical review of empirical studies. J Genet Couns. 2008;17:434-51.

23. Kasparian NA, Wakefield CE, Meiser B. Assessment of psychosocial outcomes in genetic counseling research: an overview of available measurement scales. J Genet Couns. 2007;16:693-712.

24. Skirton $\mathrm{H}$. The client's perspective of genetic counseling: a grounded theory study. J Genet Couns. 2001;10:311-29.

25. Paneque M, Sequeiros J, Skirton H. Quality issues in genetic counselling practice for presymptomatic testing: a European Delphi study. Eur J Hum Genet. 2015c;23:1468-72.

26. Lewis $L$. Models of genetic counseling and their effects on multicultura genetic counseling. J Genet Couns. 2002;11:193-212.

27. McCarthy Veach $P$, LeRoy B, Bartels D. Overview on genetic counseling history of the profession and methods of practice. In: McCarthy Veach $B$, LeRoy $B$, Bartels $D$, editors. Facilitating the genetic counseling process: a practice manual. New York: Springer- Verlag; 2003. p. 23-37.

28. McCarthy Veach $P$, Bartels D, LeRoy B. Coming full circle: a reciprocalengagement model of genetic counselling practice. J Genet Couns. 2007;16:713-28.

29. Kessler S. Psychological aspects of genetic counseling. XI. Nondirectiveness revisited. Am J Med Genet. 1997;72:164-71.

30. Farrall S, Priede C, Ruuskanen E, Jokinen A, Galev T, Arcai M, et al. Using cognitive interviews to refine translated survey questions: an example from a cross-national crime survey. Int J Soc Res Methodol. 2012;15:467-83.

31. Willis G. Cognitive interviewing as a tool for improving the informed consent process. J Empir Res Hum Res Ethics. 2006;1:9-24.

32. Almeida LS, Freire T. Metodologia da investigação em psicologia e investigação. 4ª ed. Braga: Psiquilíbrios Edições; 2009.

33. Maroco J. Análise estatística com utilização do SPSS. $3^{a}$ ed. Lisboa: Edições Sílabo; 2007.

34. Stevens J. Applied multivariate statistics for the social sciences. $4^{\text {th }}$ ed New York: Routledge; 2002.

35. Tabachnick BG, Fidell LS. Using multivariate statistics. Boston: Allyn and Bacon; 2001.

36. Paneque M, Costa C, Sequeiros J, Lemos MS. A new instrument for quality assessment of genetic counselling practice: the contribution of Portuguese genetics healthcare services. ESHG conference, Copenhague, Denmark, 2017. Eur J Hum Genet. 2017:19.46B. 